Influence of phase transition on shock-induced spallation in nanocrystalline iron

Nina Gunkelmann, Eduardo M. Bringa, and Herbert M. Urbassek’

Citation: J. Appl. Phys. 118, 185902 (2015); doi: 10.1063/1.4935452

View online: http://dx.doi.org/10.1063/1.4935452

View Table of Contents: http://aip.scitation.org/toc/jap/118/18

Published by the American Institute of Physics 


\title{
Influence of phase transition on shock-induced spallation in nanocrystalline iron
}

\author{
Nina Gunkelmann, ${ }^{1}$ Eduardo M. Bringa ${ }^{2}$ and Herbert M. Urbassek ${ }^{1, a)}$ \\ ${ }^{1}$ Physics Department and Research Center OPTIMAS, University Kaiserslautern, Erwin-Schrödinger-Straße, \\ D-67663 Kaiserslautern, Germany \\ ${ }^{2}$ CONICET and Facultad de Ciencias Exactas y Naturales, Universidad Nacional de Cuyo, Mendoza, 5500, \\ Argentina
}

(Received 7 July 2015; accepted 27 October 2015; published online 12 November 2015)

\begin{abstract}
Intense shock waves may lead to spallation of the sample. Recent experiments show differences of shock spallation in iron depending on whether the samples underwent the pressure-induced bcchcp phase transformation or not. In this study, we perform molecular dynamics simulations of shock-induced spallation in polycrystalline iron. Our results show that the phase transformation decreases the probability of multiple spallation and crack formation. In agreement with experiments, the phase transformation changes the surface morphology showing smoother spallation surfaces. (C) 2015 AIP Publishing LLC. [http://dx.doi.org/10.1063/1.4935452]
\end{abstract}

\section{INTRODUCTION}

Shock-induced phase transformations are of key interest for such diverse fields as geophysical and astronomical processes, where they provide insights into understanding meteoritic impacts, and for materials engineering, where shock-induced phase transitions may be used to harden materials resulting in a significant increase in the strength of metals. ${ }^{1}$ In particular, iron transforms from the lowpressure bcc phase (termed $\alpha$ ) at a transition pressure of around $13 \mathrm{GPa}$ to the high-pressure hcp (or $\epsilon$ ) phase. ${ }^{2,3}$ The kinetics of this transformation has been extensively studied in the past, ${ }^{4-8}$ revealing the so-called three-wave structure: The shock wave first features an elastic compression wave, then after the elastic-plastic transition, a plastic wave, and finally beyond the phase transition pressure, the wave runs in the phase-transformed material. Due to its technological and scientific importance, iron has developed into the archetypal material for studying these specific transformation shock waves. ${ }^{9-13}$ When shock waves reach the free surfaces of the target, they reflect as release waves and their superposition results in a tensile pulse. If the magnitude of the tensile stress exceeds a critical value, spall occurs. ${ }^{14}$ Note that spallation can thus only occur if specific boundary conditions are available (i.e., free back surfaces).

Recent simulations demonstrated that the phase transformation exhibits a decisive influence on the morphological changes during stress release after the shock wave. ${ }^{15}$ However, the influence of the phase transition on the spalling characteristics is still not fully understood. Most of the recent atomistic studies and experiments about spall response deal with facecentered cubic (fcc) samples. ${ }^{14,16-18}$ The effects of twin boundary spacing on spall behavior in fcc nano-twinned metals were discussed by Yuan et al. ${ }^{17}$ these authors found that twin-free $\mathrm{Ag}$ has less nucleation sources for spallation than

\footnotetext{
${ }^{a)}$ Electronic address: urbassek@rhrk.uni-kl.de. URL: http://www.physik.unikl.de/urbassek/
}

hierarchically nanotwinned Ag. Shock-induced spallation in bcc metals has been studied extensively in experiments. ${ }^{19-22}$ However, there are only a few molecular-dynamics studies on this topic. ${ }^{22-24}$

Recent experiments analyze the effects of the phase transformation on the spallation behavior of iron..$^{25,26}$ Metallurgical observations show that the morphology of the fracture surface changes in consequence of the phase transformation: while spall surfaces are rough when no phase transformation occurs, they become smoother when a phase transition was involved. ${ }^{26}$ We note that in experiment, it is possible to control the probability of phase transformation by adjusting the strain rate: high strain rates suppress the transformation. ${ }^{27}$ But a detailed understanding of the spall process from an atomistic point of view is still lacking.

In the present paper, we use MD simulations to study the propagation of shock waves through a nanocrystalline $\mathrm{Fe}$ sample with the aim of describing the interplay between the phase transformation and spallation. By comparing the results of simulations with and without phase transformation, we characterize the decisive influence of phase transformation on the spall damage.

\section{SIMULATION METHOD}

We perform our simulations under identical conditions for two established Fe potentials: the Machová and Ackland potential, ${ }^{28}$ which does not feature a phase transition at the pressures relevant for our study, and the Ackland potential ${ }^{29}$ which was designed to give the bcc $\rightarrow$ hcp transition at the experimentally observed transition pressure of $13.75 \mathrm{GPa}$. The Ackland potential is an amendment to the Machová and Ackland potential and was obtained by fitting the short-range part of the interaction to the desired transition pressure. Besides, the improved description of the bcc-hcp transition, the Ackland potential performs as well as its previous version, the Machová and Ackland potential. ${ }^{29}$ For characterization purposes, we shall denote the Machová and Ackland 
potential as the phase-stable potential, and the Ackland potential as the phase-transforming potential.

The public-domain molecular dynamics code LAMMPS (Ref. 30) was used in this paper to perform the simulations.

Our sample contains nearly 30 million atoms and has a size of $\sim 30 \times 30 \times 430 \mathrm{~nm}^{3}$. The sample was constructed with a Voronoi construction algorithm ${ }^{31}$ with an average grain size of $7.5 \mathrm{~nm}$. The complete sample contains 960 grains with more than 10 grains in the cross section.

We relaxed the sample using high-temperature annealing at $80 \%$ of the melting temperature for $100 \mathrm{ps}$; this relaxation method is necessary for equilibrating the grain boundaries (GBs). ${ }^{29}$ The shock wave simulations are conducted at a temperature of $10 \mathrm{~K}$ in order to minimize thermal noise.

Shock waves are generated by the so-called pistondriven algorithm ${ }^{32,33}$ by giving a certain particle velocity $U_{p}$ in $z$ direction to the atoms in a thin slab (thickness of one lattice constant) on one side of the sample. Note that these atoms are not subjected to the forces of the surrounding atoms. The remainder of the system volume is simulated under NVE conditions.

We consider piston velocities varying from $U_{p}=0.5$ to $U_{p}=0.9 \mathrm{~km} / \mathrm{s}$ but the main part of our discussion is devoted to $U_{p}=0.7 \mathrm{~km} / \mathrm{s}$. The piston velocity is increased linearly from 0 to the shock velocity $U_{p}$ during a ramp loading time of $15 \mathrm{ps}$. The piston moves with constant velocity during a holding time of 75 ps. Note that a sufficiently long holding time ensures that the phase-transformation to the hop structure is fully completed. We found that simulations with different holding times do not qualitatively change the results.

The piston velocity is then decreased linearly to 0 during 15 ps. Afterwards, the simulation is continued up to a total simulation time of $300 \mathrm{ps}$. We apply periodic boundary conditions in $x$ and $y$ directions and free boundaries along the shock direction.

To evaluate the results, we determined various material properties in dependence of the $z$ position in the polycrystal. These profiles were determined by dividing the sample into small regions. Each of these equally sized regions is a small slab with a thickness of $0.8565 \mathrm{~nm}$ along the $z$ direction. We calculate the velocity profile in $z$ direction, $v_{z}$, the stress in $z$ direction, $p_{z z}$, the relative density, $\phi$, the temperature, $T$, and the shear stress, $p_{\text {shear }}$. The latter is defined as

$$
p_{\text {shear }}=\frac{1}{2}\left(p_{z z}-p_{\text {trans }}\right),
$$

where $p_{i j}$ denote the components of the stress tensor, and the transverse stress is defined as

$$
p_{\text {trans }}=\frac{1}{2}\left(p_{x x}+p_{y y}\right) .
$$

We measure the relative density $\phi$ by normalizing the atom density in each slab to the bulk density of iron.

For visualization of the atomistic configurations, we use common-neighbor analysis (CNA) $)^{34,35}$ and the adaptive common-neighbor analysis ${ }^{36}$ within the free software tool OVITO. ${ }^{37}$ We also used the Crystal Analysis Tool (CAT) ${ }^{36}$ to detect bec twins.

\section{RESULTS}

For a qualitative overview, we display in Figs. 1 and 2 the changes induced in our sample by the passage of the shock and release wave. For the following simulations, we used a piston velocity of $U_{p}=0.7 \mathrm{~km} / \mathrm{s}$.

In Fig. 1, the part of the sample, where the spall occurs, is displayed. Fig. 1(a) shows the results for the phase-stable Machová and Ackland potential. During recovery of the sample at around $100 \mathrm{ps}$, bcc twins nucleate at the grain boundaries. At $140 \mathrm{ps}$, the spallation process has started; it is visible as a series of small voids near the grain boundaries at the surface. Later, we observe amorphization of the intergranular regions where the voids were nucleated. ${ }^{38,39}$ No voids are formed in the grain interior. A small fraction of fcc phase nucleates but vanishes almost completely again at later times. At $160 \mathrm{ps}$, the spall region is molten (visible by the dissolution of crystalline grains and the appearance of a continuous defect zone). The spall is complete at $180 \mathrm{ps;}$ the spall surfaces are rough.

For the Ackland potential, which allows for the pressure-induced transformation, the situation has qualitatively changed considerably, see Fig. 1(b). At 100 ps, the phase transformation to hcp is almost fully completed; embedded in the hcp grains, a number of fcc stacking faults are observed. Due to stress release, the sample transforms back

(a)
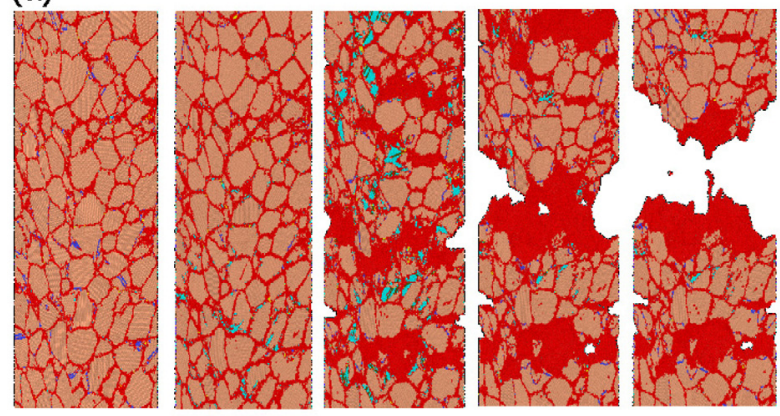

(b)

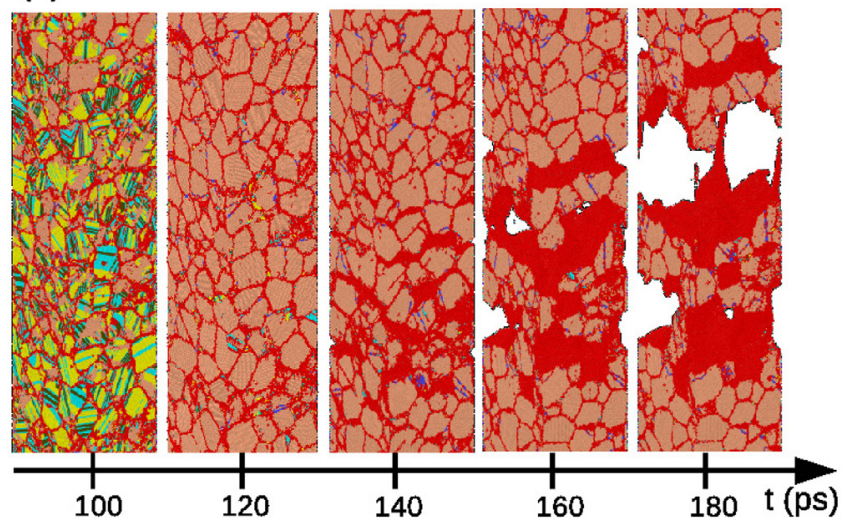

FIG. 1. Snapshots of a region of the sample for the (a) phase-stable Machová and Ackland and the (b) phase-transforming Ackland potential. The region extends between (a) 60 and $515 \mathrm{~nm}$ and (b) 60 and $480 \mathrm{~nm}$ from the position where the shock-wave starts. Shock wave runs upward. Local atomic structures are identified by adaptive CNA and CAT. ${ }^{36}$ Pink: bcc; red: bcc vacancies and intergranular atoms; yellow: hcp; teal: fcc; blue: bcc twin boundary. 


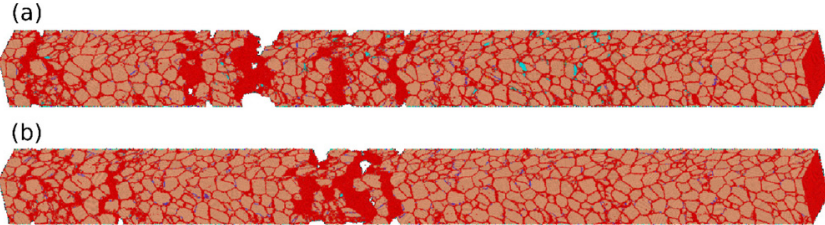

FIG. 2. Snapshots of the sample at $160 \mathrm{ps}$ after start of the shock wave for the (a) phase-stable Machová and Ackland and the (b) phase-transforming Ackland potential. Shock wave runs from left to right. Local atomic structures are identified by adaptive CNA and CAT ${ }^{36}$ Pink: bcc; red: bcc vacancies and intergranular atoms; yellow: hcp; teal: fcc; blue: bcc twin boundary.

to bcc; this reverse phase transition to bcc is finished at 120 ps. At this time, several morphological features are observed, such as dislocations and bcc twins; this is characteristic of the stress release stage. ${ }^{15}$ Note that the amount of bcc twins at $140 \mathrm{ps}$ is considerably higher than for the Machová and Ackland potential indicating that the phase transformation helps to drive twinning. ${ }^{15}$ At 140 ps, we see first cracks and voids which indicate the beginning of spall. Twin boundaries are created and destroyed in that region where the spall is going to occur. However, the amount of bcc twin boundaries remains roughly constant (see Fig. 5 below). These twins survive recovery and provide sources for void nucleation. The spall occurs somewhat later than for the Machová and Ackland potential.

To compare the entire morphology of the spalling specimens, Fig. 2 shows the entire samples at 160 ps after start of the shock wave for the two simulations. For the case without phase transition, we observe many cracks, leading to multispallation, while the phase-transforming sample only exhibits a concentrated region where spallation is visible. These differences in crack occurrence and frequency will show up later repeatedly, such as in the relative density and the temperature profiles, Figs. 6(d) and 6(e). Fig. 3 visualizes the stress component in shock direction $p_{z z}$ for the samples at 160 ps after start of the shock wave for the two potentials. At this time, the shock wave was already reflected from the right-hand surface and stresses are tensile $\left(p_{z z}<0\right)$ throughout the specimen, see Fig. 6(b) below. The peak amplitude of the tensile stress initiating spall already passed through the cracked regions, such that the background stress is there near zero. Note, however, the localized tensile stress peaks immediately at the crack zones; these are induced by strongly strained bonds which are about to break and thus highlight the ongoing crack process.

Fig. 4 gives a close-up view of the spalled surfaces. The surface is more brittle for the Machova and Ackland potential where parts of the surface are dissolved. For quantitative

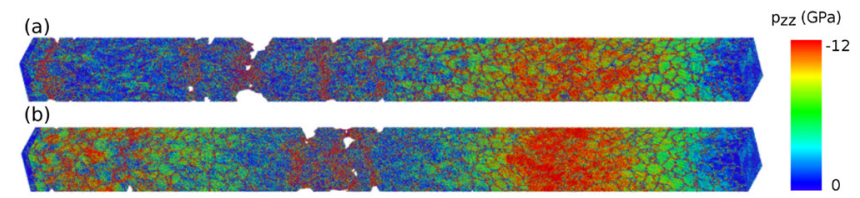

FIG. 3. Snapshots of the sample at $160 \mathrm{ps}$ after start of the shock wave for the (a) phase-stable Machová and Ackland and the (b) phase-transforming Ackland potential. Shock wave runs from left to right. The color codes the stress component in shock direction, $p_{z z}$.
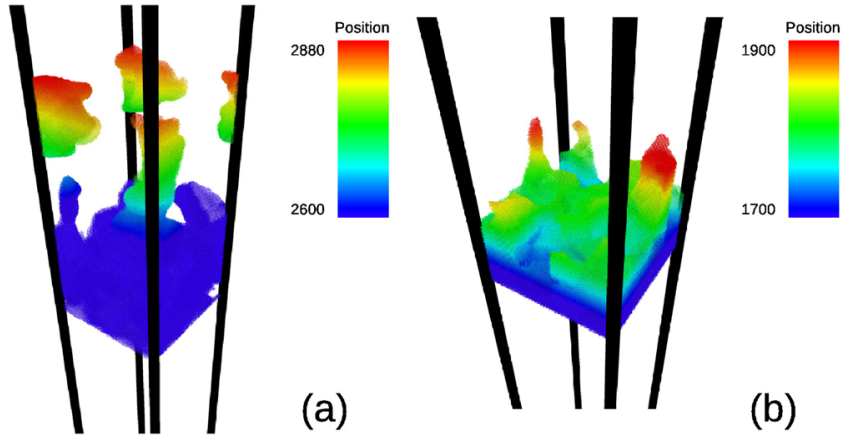

FIG. 4. Spall surface for the (a) phase-stable Machová and Ackland and the (b) phase-transforming Ackland potential. The color codes the $z$ position in the crystal in units of $1 \times 10^{-10} \mathrm{~m}$.

analysis of the surface roughness, we relax the spalled samples at the end of the simulation at 300 ps in a Nosé/Hoover isenthalpic $(\mathrm{NpH})$ ensemble and cool back to $10 \mathrm{~K}$ during a total time of $100 \mathrm{ps}$. This relaxation process is necessary because the surfaces are molten. The surface roughness $R_{q}$ is quantified by the root-mean-square average of the profile height deviations from the mean. For the Ackland potential, we measure $R_{q}=2.17 \mathrm{~nm}$ and for the Machová and Ackland potential, we find $R_{q}=2.73 \mathrm{~nm}$. The higher roughness of the non-phase-transformed surface is in qualitative agreement with experimental results by de Rességuier et $a .^{26}$ who observed that the occurrence of the reverse phase transformation leads to smoother fracture surfaces.

Fig. 5 shows the evolution of the twin fraction-defined as the number of atoms forming twin boundaries with respect to the total number of atoms-versus time for both potentials. As expected, the number of twins is higher for the potential which allows for phase transition because twin growth is intimately related to the phase transformation as shown in Ref. 15. For the Machová and Ackland potential, the number of twins decreases during release but increases after spallation due to the high shear stresses in the sample. For both potentials, the number of twins eventually reaches a constant value. As de Rességuier et al. ${ }^{26}$ noted, a dense distribution of twin boundaries could act as activation sites for void nucleation, resulting in smooth macroscopic fracture located in a narrow zone. This fact might explain the

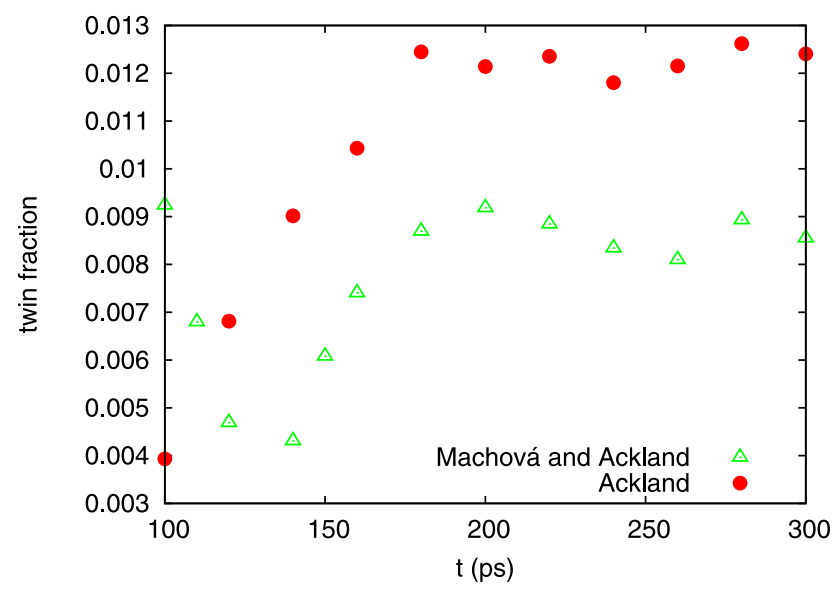

FIG. 5. Twin fraction versus time. 

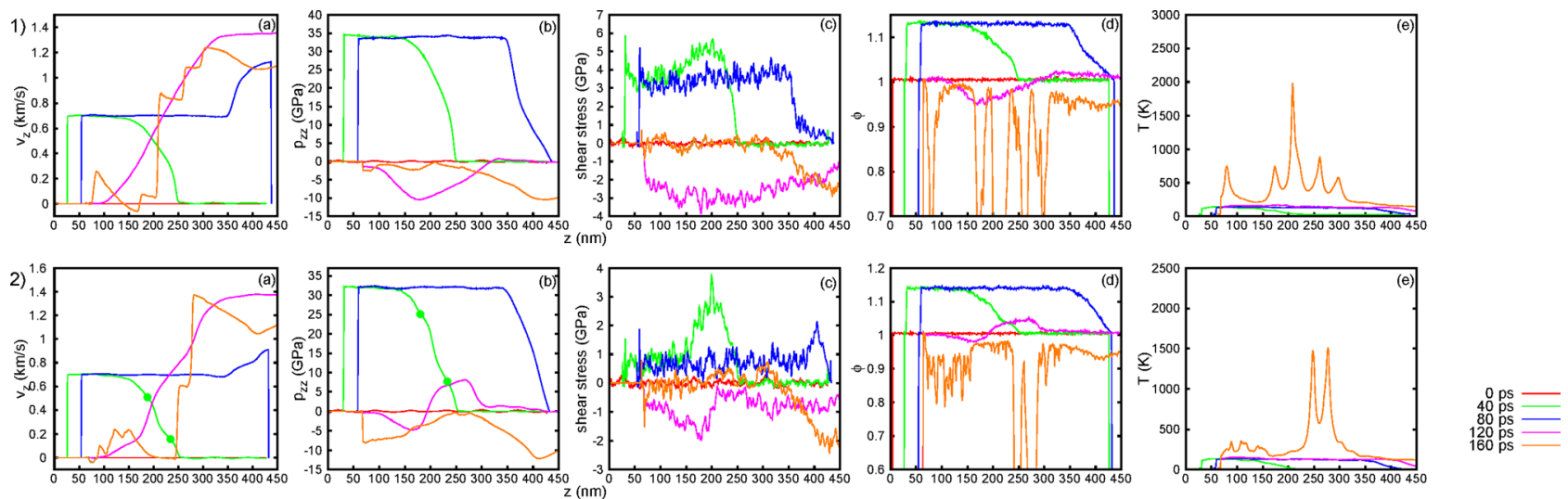

FIG. 6. Spatial profiles of the (a) atom velocity in $z$-direction, $v_{z}$, the (b) stress components parallel to the shock wave propagation direction, $p_{z z}$, (c) shear stress, Eq. (1), (d) local filling factor, and (e) temperature. The simulation is performed for the Machová and Ackland potential (top row: 1) and the Ackland potential (bottom row: 2). The so-called knees appearing in $v_{z}$ and $p_{z z}$ in the Ackland profiles are marked by dots.

morphological differences observed for the spall surface in the two potentials. In our simulation, we observe void nucleation primarily at GBs, but twins caused by recovery of the phase transition could also provide void nucleation sites. It was shown in simulations for $\mathrm{Ni}$ (Ref. 40) that shockinduced boundaries would facilitate void nucleation. Recent experiments in Ta also show void nucleation inside grains, due to defects. ${ }^{41}$ Hahn et $a l .{ }^{42}$ observed voids nucleated at twin-twin intersections. In our simulations, we do not have a sufficiently large amount of twinning to allow for twin-twin intersections.

We evaluate the dynamics within the shock and release wave by showing its spatially resolved characteristics at various times (Fig. 6). The results for the Ackland potential are identical to a previous study, ${ }^{7}$ showing two inflection points (the so called knees) in the velocity, $v_{z}(z)$, and stress, $p_{z z}(z)$, profiles. These "knees" directly indicate the three-wave structure: An elastic precursor wave is followed by a plastic wave which then leads to a phase-transformation front. The spall signal is identified by a sudden drop in the velocity profile. It starts at around $120 \mathrm{ps}$ for the phase-transforming potential visible as a kink at $260 \mathrm{~nm}$ in Fig. 6(a). For the Machová and Ackland potential, spallation starts slightly later at $130 \mathrm{ps}$ at $210 \mathrm{~nm}$. Note that we also performed simulations for a holding time of $35 \mathrm{ps}$ with piston speeds of $U_{p}=0.5$ and $0.9 \mathrm{~km} / \mathrm{s}$; these gave spall times of 106.0 and 102.3 ps, respectively, showing that the spall time decreases slightly for higher piston velocities. This is the trivial consequence of the increase in wave speed with piston speed. Apart from these changes, simulations with different piston velocities do not qualitatively change the results. The spall stress was identified as the minimum value of $p_{z z}$; it amounts to $\sim 13.6 \mathrm{GPa}$ for the phasetransforming potential and $\sim 12.4 \mathrm{GPa}$ for the phase-stable potential. These values are comparable to the experimental tensile stress before fracture of $8 \mathrm{GPa}$ measured in thin iron foils of $150 \mu \mathrm{m}$ thickness. ${ }^{26}$ In this study, the authors observe higher spall stresses after the bcc-hcp-bcc transformation cycle consistent with our finding.

Fig. 6(c) shows the shear stress for both potentials. For the Ackland potential, we observe a decrease in shear stress at
$80 \mathrm{ps}$ and $370 \mathrm{~nm}$ associated with the phase transformation. ${ }^{7,8}$ The phase-stable Machová and Ackland potential exhibits constant shear stress and no indication of plasticity can be seen before unloading. The maximum spall shear stress is $3 \mathrm{GPa}$ for the Machová and Ackland potential and 2.2 GPa for the Ackland potential.

The cracks can also be detected by measuring the relative density, Fig. 6(d). Here, we observe decidedly more cracks for the Machová and Ackland potential than for the Ackland potential.

Finally, the spall signal also appears in the temperature profile, Fig. 6(e). The average temperature is significantly higher for the phase-stable potential because the formation of cracks and voids dissipates energy and becomes visible as maxima in the temperature profile. For the phase-stable potential, the temperature even rises above the melting temperature $(1811 \mathrm{~K})$ at the spall surfaces.

From the velocity profiles, we evaluate the velocity of the free surface versus time. Fig. 7 shows the back surface velocity versus time for both potentials, and displays a clear pull-back signal as observed in typical spall experiments. Spall starts at about $130 \mathrm{ps}$, but the pull-back signal appears in the back surface at around 175 ps, which is expected given our sample thickness.

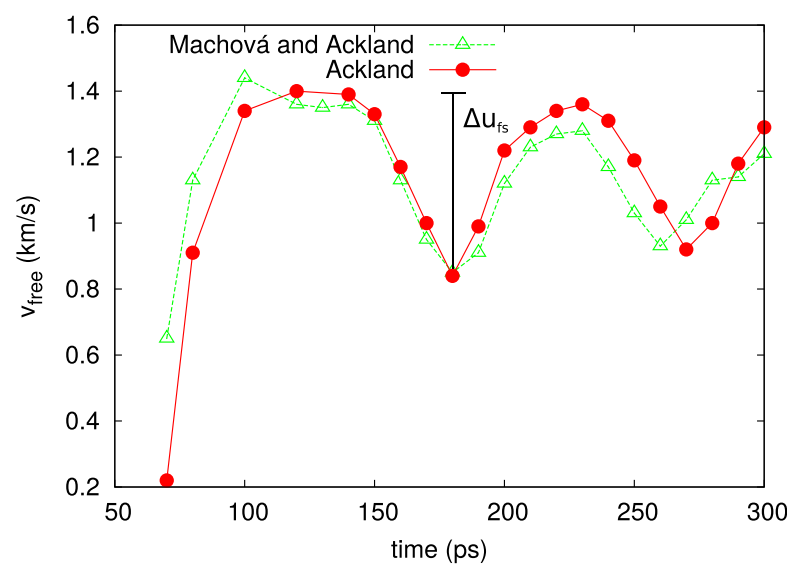

FIG. 7. Free-surface velocity versus time for the phase-stable Machová and Ackland and for the phase-transforming Ackland potentials. 
Note that the spall stress can also be calculated from the free-surface velocity. We use the following linear approximation: ${ }^{43}$

$$
p_{\text {spall }}=\frac{1}{2} \rho_{0} c_{0} \Delta u_{\mathrm{fs}},
$$

where $\rho_{0}=7874 \mathrm{~kg} / \mathrm{m}^{3}$ is the initial density, $c_{0}=5170 \mathrm{~m} / \mathrm{s}$ is the sound velocity, and $\Delta u_{\mathrm{fs}} \sim 600 \mathrm{~m} / \mathrm{s}$ is the pullback-signal defined as the difference between the peak free-surface velocity and the minimum free-surface velocity ahead of the spall pulse (see Fig. 7). The resulting spall stress is $\sim 12.2 \mathrm{GPa}$ for both potentials and roughly agrees with our moleculardynamics result which was 12.4 and $13.6 \mathrm{GPa}$ for the phasestable and phase-transforming potentials, respectively.

We note that the pullback signals plotted in Fig. 7 are quite similar. This is due to the extremely short (compared to experiment) shock pulse reaching the rear surface. Since the pullback signal is the simple consequence of the reflection of a wave, its character does not change much for such short pulses. This is, in particular, true if the sample cross section is small and the spall occurs at the same position. In contrast, the roughness of the spalled surface is governed by materials properties, such that the same pullback signal may result in different surface morphologies if the interatomic interactions are different. There exist several MD studies which evaluate pullback signals. ${ }^{23,24,44,45}$ Gray et al. ${ }^{46}$ observed that shockloaded samples with three different peak shock stresses displayed nearly identical pullback signals although the damage fields were different. This appears plausible since at high strain rates, the rate of plastic relaxation is slower than the rate at which the tensile stress develops.

The decay of the oscillations in Fig. 7 is a consequence of the damping of the waves due to viscosity in the liquid. We observe that the wave travels faster for the phase-transforming potential in agreement with experiments by Chen et al. ${ }^{25}$

\section{CONCLUSIONS}

In this paper, we present large-scale shock and release simulations in polycrystalline iron in order to analyze the effects of the pressure-induced bcc $\rightarrow \mathrm{cp}$ phase transition on spallation. Spall damage is found to be strongly affected by the phase transition, leading to a changed morphology of the fracture surface, in agreement to experiment.

In detail we find the following.

(1) The phase transformation decreases the probability of multiple spallation and crack formation.

(2) The fracture surface is influenced by the phase transition showing smooth spall surfaces.

(3) Twin growth is driven by the phase transformation. The twins may provide sources for void nucleation and may explain the smooth fracture surfaces.

\section{ACKNOWLEDGMENTS}

This work has been supported by the Deutsche Forschungsgemeinschaft via the Sonderforschungsbereich
926. E.M.B. thanks support from a project SeCTyP-UNCuyo. Simulations were performed at the High Performance Cluster Elwetritsch (RHRK, TU Kaiserslautern, Germany). We thank E. Hahn for useful comments.

${ }^{1}$ N. K. Bourne, Materials in Mechanical Extremes: Fundamentals and Applications (Cambridge University Press, Cambridge, 2013).

${ }^{2}$ P. M. Giles, M. H. Longenbach, and A. R. Marder, J. Appl. Phys. 42, 4290 (1971).

${ }^{3} \mathrm{~W}$. Pepperhoff and M. Acet, Constitution and Magnetism of Iron and its Alloys (Springer, Berlin, 2001).

${ }^{4}$ K. Kadau, T. C. Germann, P. S. Lomdahl, and B. L. Holian, Science 296, 1681 (2002).

${ }^{5}$ K. Kadau, T. C. Germann, P. S. Lomdahl, and B. L. Holian, Phys. Rev. B 72, 064120 (2005).

${ }^{6}$ K. Kadau, T. C. Germann, P. S. Lomdahl, R. C. Albers, J. S. Wark, A. Higginbotham, and B. L. Holian, Phys. Rev. Lett. 98, 135701 (2007).

${ }^{7}$ N. Gunkelmann, E. M. Bringa, D. R. Tramontina, C. J. Ruestes, M. J. Suggit, A. Higginbotham, J. S. Wark, and H. M. Urbassek, Phys. Rev. B 89, 140102 (2014).

${ }^{8}$ N. Gunkelmann, D. R. Tramontina, E. M. Bringa, and H. M. Urbassek, New J. Phys. 16, 093032 (2014).

${ }^{9}$ L. M. Barker and R. E. Hollenbach, J. Appl. Phys. 45, 4872 (1974).

${ }^{10}$ D. H. Kalantar, J. F. Belak, G. W. Collins, J. D. Colvin, H. M. Davies, J. H. Eggert, T. C. Germann, J. Hawreliak, B. L. Holian, K. Kadau et al., Phys. Rev. Lett. 95, 075502 (2005).

${ }^{11}$ B. J. Jensen, G. T. Gray III, and R. S. Hixson, J. Appl. Phys. 105, 103502 (2009).

${ }^{12}$ J. A. Hawreliak, B. El-Dasher, H. Lorenzana, G. Kimminau, A. Higginbotham, B. Nagler, S. M. Vinko, W. J. Murphy, T. Whitcher, J. S. Wark et al., Phys. Rev. B 83, 144114 (2011).

${ }^{13}$ R. F. Smith, J. H. Eggert, D. C. Swift, J. Wang, T. S. Duffy, D. G. Braun, R. E. Rudd, D. B. Reisman, J.-P. Davis, M. D. Knudson et al., J. Appl. Phys. 114, 223507 (2013).

${ }^{14}$ V. Dremov, A. Petrovtsev, P. Sapozhnikov, M. Smirnova, D. L. Preston, and M. A. Zocher, Phys. Rev. B 74, 144110 (2006).

${ }^{15}$ N. Gunkelmann, D. R. Tramontina, E. M. Bringa, and H. M. Urbassek, J. Appl. Phys. 117, 085901 (2015).

${ }^{16}$ J. P. Escobedo, E. N. Brown, C. P. Trujillo, E. K. Cerreta, and G. Gray, J. Appl. Phys. 113, 103506 (2013).

${ }^{17}$ F. Yuan, L. Chen, P. Jiang, and X. Wu, J. Appl. Phys. 115, 063509 (2014).

${ }^{18}$ S. J. Fensin, S. M. Valone, E. K. Cerreta, J. P. Escobedo-Diaz, G. T. Gray III, K. Kang, and J. Wang, Modell. Simul. Mater. Sci. Eng. 21, 015011 (2013).

${ }^{19}$ A. K. Zurek, W. R. Thissell, J. N. Johnson, D. L. Tonks, and R. Hixson, J. Mater. Process. Technol. 60, 261 (1996).

${ }^{20}$ G. I. Kanel, S. V. Razorenov, A. V. Utkin, V. E. Fortov, K. Baumung, H. U. Karow, D. Rusch, and V. Licht, J. Appl. Phys. 74, 7162 (1993).

${ }^{21}$ S. I. Anisimov, N. A. Inogamov, Y. V. Petrov, V. A. Khokhlov, V. V. Zhakhovskii, K. Nishihara, M. B. Agranat, S. I. Ashitkov, and P. S. Komarov, Appl. Phys. A 92, 797 (2008).

${ }^{22}$ Q. An, R. Ravelo, T. C. Germann, W. Z. Han, S. N. Luo, D. L. Tonks, and W. A. Goddard III, AIP Conf. Proc. 1426, 1259 (2012).

${ }^{23}$ J.-P. Cuq-Lelandais, M. Boustie, L. Soulard, L. Berthe, J. Bontaz-Carion, and T. de Resseguier, AIP Conf. Proc. 1426, 1167 (2012).

${ }^{24}$ J.-P. Cuq-Lelandais, M. Boustie, L. Soulard, L. Berthe, A. Solliei, J. Bontaz-Carion, P. Combis, T. de Resseguier, and E. Lescoute, AIP Conf. Proc. 1195, 829 (2009).

${ }^{25}$ Y. Chen, X. Tang, and Q. Li, Chin. Phys. B 19, 056402 (2010).

${ }^{26}$ T. de Rességuier and M. Hallouin, Phys. Rev. B 77, 174107 (2008).

${ }^{27}$ J. C. Crowhurst, B. W. Reed, M. R. Armstrong, H. B. Radousky, J. A. Carter, D. C. Swift, J. M. Zaug, R. W. Minich, N. E. Teslich, and M. Kumar, J. Appl. Phys. 115, 113506 (2014).

${ }^{28}$ A. Machová and G. J. Ackland, Modell. Simul. Mater. Sci. Eng. 6, 521 (1998).

${ }^{29}$ N. Gunkelmann, E. M. Bringa, K. Kang, G. J. Ackland, C. J. Ruestes, and H. M. Urbassek, Phys. Rev. B 86, 144111 (2012).

${ }^{30}$ S. Plimpton, J. Comput. Phys. 117, 1 (1995).

${ }^{31}$ A. Frøseth, H. Van Swygenhoven, and P. Derlet, Acta Mater. 53, 4847 (2005).

${ }^{32}$ B. L. Holian and P. S. Lomdahl, Science 280, 2085 (1998). 
${ }^{33}$ E. M. Bringa, K. Rosolankova, R. E. Rudd, B. A. Remington, J. S. Wark, M. Duchaineau, D. H. Kalantar, J. Hawreliak, and J. Belak, Nature Mater. 5, 805 (2006)

${ }^{34}$ D. Faken and H. Jonsson, Comput. Mater. Sci. 2, 279 (1994).

${ }^{35}$ H. Tsuzuki, P. S. Branicio, and J. P. Rino, Comput. Phys. Commun. 177, 518 (2007)

${ }^{36}$ A. Stukowski, Modell. Simul. Mater. Sci. Eng. 20, 045021 (2012).

${ }^{37}$ A. Stukowski, Modell. Simul. Mater. Sci. Eng. 18, 015012 (2010).

${ }^{38}$ Y. Ashkenazy and R. S. Averback, Appl. Phys. Lett. 86, 051907 (2005).

${ }^{39}$ S. G. Srinivasan, M. I. Baskes, and G. J. Wagner, J. Appl. Phys. 101, 043504 (2007).

${ }^{40}$ S. Srinivasan, M. Baskes, and G. Wagner, J. Mater. Sci. 41, 7838 (2006).
${ }^{41}$ J. Escobedo, E. Cerreta, and D. Dennis-Koller, J. Miner. Met. Mater. Soc. 66, 156 (2014).

${ }^{42}$ E. N. Hahn, T. C. Germann, R. J. Ravelo, J. E. Hammerberg, and M. A. Meyers, in 19th Biennial Conference on Shock Compression of Condensed Matter, 2015 (Tampa, FL, submitted).

${ }^{43}$ S. A. Novikov, I. I. Divnov, and A. G. Ivanov, Phys. Metals Metallogr. 21, 4 (1966).

${ }^{44}$ S.-N. Luo, Q. An, T. C. Germann, and L.-B. Han, J. Appl. Phys. 106, 013502 (2009).

${ }^{45}$ S.-N. Luo, T. C. Germann, and D. L. Tonks, J. Appl. Phys. 106, 123518 (2009).

${ }^{46}$ G. T. Gray III, N. K. Bourne, V. Livescu, C. P. Trujillo, S. MacDonald, and P. Withers, J. Phys.: Conf. Ser. 500, 112031 (2014). 